\title{
A computational modeling for real ecosystems based on P systems
}

\author{
Mónica Cardona $\cdot$ M. Angels Colomer • Antoni Margalida • Antoni Palau $\cdot$ Ignacio \\ Pérez-Hurtado • Mario J. Pérez-Jiménez • Delfí Sanuy
}

\begin{abstract}
In this paper, a P systems based general framework for modeling ecosystems dynamics is presented. Particularly, ecosystems are specified by means of multienvironment $\mathrm{P}$ systems composed of a finite number of environments, each of them having an extended $\mathrm{P}$ system with active membranes. The semantics is of a probabilistic type and it is implemented by assigning each rule of the system a probabilistic constant which depends on the environment and the run time. As a case study, two real ecosystems are described: scavenger birds in the Catalan Pyrenees and the zebra mussel (Dreissena Polymorpha) in Ribarroja reservoir (Spain).
\end{abstract}

M. Cardona - M. A. Colomer $(\bowtie)$

Department of Mathematics, University of Lleida, Av. Alcalde Rovira Roure, 191, 25198 Lleida, Spain e-mail: colomer@matematica.udl.es

M. Cardona

e-mail: mcardona@matematica.udl.es

A. Margalida

Bearded Vulture Study \& Protection Group, Adpo. 43, 25520 El Pont de Suert (Lleida), Spain e-mail: margalida@inf.entorno.es

A. Palau

Dirección de Medio Ambiente y Desarrollo Sostenible, Endesa, Spain

e-mail: antonio.palau@endesa.es

I. Pérez-Hurtado · M. J. Pérez-Jiménez

Research Group on Natural Computing, Department of Computer Science and Artificial Intelligence, University of Sevilla, Avda. Reina Mercedes s/n, 41012 Sevilla, Spain

e-mail: perezh@us.es

M. J. Pérez-Jiménez

e-mail: marper@us.es

D. Sanuy

Department of Animal Production, University of Lleida, Av. Alcalde Rovira Roure, 191, 25198 Lleida, Spain

e-mail: dsanuy@prodan.udl.cat 
Keywords P-System · Ecosystem $\cdot$ Probabilistic $\cdot$ Dynamics $\cdot$ Scavenger $\cdot$ Mussel

\section{Introduction}

In the evolution of an ecosystem, a high number of factors are involved, even having a dynamical interconnection. This makes the modeling process of an ecosystem a hard task, and consequently, it is necessary to delimit the problem by setting the values some of variables involved in the design.

Volterra and Lotka proposed the first models of population dynamics based on systems of differential equations: the predator-prey model with resources limitations for the prey (Volterra 1926a, b) and the model of chemical reactions in which chemical concentrations oscillate (Lotka 1925). From them, it is obtained a system of ordinary differential equations which generalizes both models and is referred to as the Lotka-Volterra model. A P systems based algorithm (Fontana and Manca 2008) has already been used to analize predator-prey dynamics expressed in the traditional formulation by Lotka and Volterra.

In contrast to differential equations, $\mathrm{P}$ systems explicitly correspond to the discrete character of the components of an ecosystem and use rewriting rules on multisets of objects which represent the variables of the system. The inherent stochasticity, external noise and uncertainty in cellular systems is captured by using probabilistic strategies.

The $\mathrm{P}$ systems based modeling of an ecosystem allows us to study the simultaneous evolution of a high number of species. Furthermore, modularity in this P systems enables the addition of new ingredients in a quite simple way. In this paper, a $\mathrm{P}$ systems based general framework for modeling ecosystems dynamics is presented. Ecosystems are described by means of multienvironment $P$ systems consisting of a finite number of environments, each of them having a specific $P$ system with active membranes. Each rule has associated a real number from $[0,1]$ which depends on the left-hand side of the rule and the run time.

As a case study, two real ecosystems are described: scavenger birds in the Catalan Pyrenees and the zebra mussel in Ribarroja reservoir (Spain). The most interesting aspect of the former is the possibility to predict the evolution of endangered species under different scenarios. In this way, it is possible to find out what is the most adequate management of the ecosystem for the sustainable development of species. The aim of the zebra mussel modeling is the design of a management strategy for the reservoir in order to control, or eradicate if possible, the population of an invasive species (zebra mussel) which causes serious environmental and economic damages.

The paper is organized as follows. The next section shows a P systems based general framework for modeling ecosystems dynamics. In Sect. 3, we present a software tool which allows us to simulate the ecosystems behavior under different scenarios. Section 4 is devoted to the study of an ecosystem related to scavenger birds at the Catalan Pyrenees, and the results obtained by using a previous model are presented (Cardona et al. 2009a, b). In Sect. 5, the first model of an ecosystem related to the zebra mussel at Ribarroja reservoir is presented. That model has not been validated experimentally yet. Finally, some conclusions and future work are discussed in Sect. 6.

\section{A P system based model of an ecosystem}

First, we define a new P systems based framework (probabilistic P systems) where additional features, such as electrical charges which describe specific properties in a better way, are used. 
Definition 1 A skeleton of an extended P system with active membranes of degree $q \geq 1$ is a tuple $\Pi=(\Gamma, \mu, R)$ where:

- $\quad \Gamma$ is an alphabet (the working alphabet);

- $\quad \mu$ is a membrane structure consisting of $q$ membranes, with the membranes labeled $0,1, \ldots, q-1$ in a one-to-one way with membranes. The skin membrane is labeled with 0 . We also associate electrical charges out of the set $\{0,+,-\}$ with membranes;

- $R$ is a finite set of evolution rules of the form $r: u[v]_{i}^{\alpha} \rightarrow u^{\prime}\left[v^{\prime}\right]_{i}^{\alpha^{\prime}}$ where $u, v, u^{\prime}, v^{\prime}$ are multisets over $\Gamma), i \in\{0,1, \ldots, q-1\}$, and $\alpha, \alpha^{\prime} \in\{0,+,-\}$;

A skeleton of an extended $\mathrm{P}$ system with active membranes of degree $q \geq 1, \Pi=$ $(\Gamma, \mu, R)$, can be viewed as a set of (polarizated) membranes hierarchized by a structure $\mu$ and labeled by $0,1, \ldots, q-1$. All membranes in $\mu$ are supposed to be (initially) neutral and they have associated a finite set $R$ of evolution rules that can modify their polarization.

Definition 2 A probabilistic functional extended $\mathrm{P}$ system with active membranes of degree $q \geq 1$ taking $T$ time units is a tuple

$$
\Pi=\left(\Gamma, \mu, R, T,\left\{f_{r}: r \in R\right\}, \mathcal{M}_{0}, \ldots, \mathcal{M}_{q-1}\right)
$$

where:

- $\quad(\Gamma, \mu, R)$ is the skeleton of an extended $\mathrm{P}$ system with active membranes of degree $q$

- $\quad T$ is a natural number, $T \geq 1$;

- $f_{r}, r \in R$, is a computable function such that $\operatorname{dom}\left(f_{r}\right) \subseteq\{1, \ldots, T\}$, and range $\left(f_{r}\right) \subseteq$ $[0,1]$ verifying the following:

If $r_{1}, \ldots, r_{z}$ are the rules from $R$ with the same left-hand side (i.e. $u[v]_{i}^{\alpha}$ then $\sum_{j=1}^{z} f_{r}(a)=1$, for $a=1,2, \ldots, T$.

- $\mathcal{M}_{0}, \ldots, \mathcal{M}_{q-1}$ are strings over $\Gamma$, describing the multisets of objects initially placed in the $q$ regions of $\mu$.

A probabilistic functional extended $\mathrm{P}$ system with active membranes of degree $q \geq 1$ taking $T$ time units, $\Pi=\left(\Gamma, \mu, R, T,\left\{f_{r}: r \in R\right\}, \mathcal{M}_{0}, \ldots, \mathcal{M}_{q-1}\right)$, can be viewed as a set of membranes hierarchized by the structure $\mu$ labeled by $0,1, \ldots, q-1$. T is a natural number that represents the simulation time of the system. For each $r \in R$ and $a=1,2, \ldots, T, f_{r}(a)$ represents a probabilistic constant associated with rule $r$ at moment $t$. In a generic way, we denote $r: u[v]_{i}^{\alpha} \stackrel{f_{r}(a)}{\longrightarrow} u^{\prime}\left[v^{\prime}\right]_{i}^{\alpha^{\prime}}$. If $f_{r}(a)=1$, then we briefly denote $r: u[v]_{i}^{\alpha} \longrightarrow u^{\prime}\left[v^{\prime}\right]_{i}^{\alpha^{\prime}}$.

The $q$-tuple of multisets present at a given moment in the $q$ regions of the system constitutes the configuration of the system at that moment. The $q$-tuple $\left(\mathcal{M}_{0}, \ldots, \mathcal{M}_{q-1}\right)$ is the initial configuration of the system $\Pi$.

The $\mathrm{P}$ system can pass from one configuration to another by applying the rules from $R$ as follows:

- A rule $u[v]_{i}^{\alpha} \rightarrow u^{\prime}\left[v^{\prime}\right]_{i}^{\alpha^{\prime}}$ is applicable to a membrane labeled by $i$, and with $\alpha$ as electrical charge if multiset $u$ is contained in the father of membrane $i$, and multiset $v$ is contained 
in the membrane labeled by $i$ having $\alpha$ as electrical charge. When that rule is applied, multiset $u$ (resp. $v$ ) in the father of membrane $i$ (resp. in membrane $i$ ) is removed from that membrane, and multiset $u^{\prime}$ (resp. $v^{\prime}$ ) is produced in that membrane, changing its label to $\alpha^{\prime}$.

- The rules of the system are applied in a maximal consistent parallel way; that is, for each $i \in\{0,1, \ldots, q-1\}, \alpha, \alpha^{\prime} \in\{0,+,-\}$ all applicable rules of the type $u[v]_{i}^{\alpha} \rightarrow$ $u^{\prime}\left[v^{\prime}\right]_{i}^{\alpha^{\prime}}$, for $u, v, u^{\prime}, v^{\prime}$ multisets over $\Gamma$, must be simultaneously applied in a maximal parallel way.

- For each $u, v$ multisets over $\Gamma, i \in\{0, \ldots, q-1\}$ and $\alpha \in\{0,+,-\}$, if $r_{1}, \ldots, r_{z}$ are the rules applicable whose left-hand side is $u[v]_{i}^{\alpha}$ in at given moment $a$, then rules will be applied according to the corresponding probabilities $f_{r_{1}}(a), \ldots, f_{r_{z}}(a)$, that is, if these rules compete for $K$ blocks of objects (i.e. in membrane $i$ there is $v^{k}$ and in its father there is $u^{k}$ ), then these blocks are distributed according to the probabilities associated with the rules.

Definition 3 A multienvironment probabilistic functional extended $\mathrm{P}$ system with active membranes of degree $(q, m)$ with $q \geq 1, m \geq 1$, taking $T$ time units, $T \geq 1$, is a tuple

$$
\left(\Gamma, \Sigma, G, R_{E}, \Pi,\left\{f_{r, j}: r \in R_{\Pi}, 1 \leq j \leq m\right\},\left\{M_{i j}: 0 \leq i \leq q-1,1 \leq j \leq m\right\}\right)
$$

where:

- $\quad \Gamma$ is the working alphabet and $\Sigma \varsubsetneqq \Gamma$ is an alphabet representing the objects that can be present in the environments;

- $G=(V, S)$ is a directed graph such that $(e, e) \in S$, for each $e \in V$. Let $V=\left\{e_{1}, \ldots, e_{m}\right\}$ whose elements are called environments;

- $R_{E}$ is a finite set of communication rules of the form

$$
(x)_{e_{j}} \stackrel{p_{\left(x, j, j^{\prime}\right)}}{\longrightarrow}(y)_{e_{j^{\prime}}}
$$

where $x, y \in \Sigma,\left(e_{j}, e_{j^{\prime}}\right) \in S, 1 \leq j, j^{\prime} \leq m$, and $p_{\left(x, j, j^{\prime}\right)}$ is a computable function whose domain is $\{1,2, \ldots, T\}$ and its range is contained in $[0,1]$. These rules verify the following: for each $e_{j}$, if $\left\{e_{j_{1}}, \ldots e_{j_{z}}\right\}$ is the set of reachable nodes from $e_{j}$, then $\sum_{i=1}^{z} p_{\left(x, j, j_{i}\right)}(a)=1$, for each $x \in \Sigma$ and $a=1,2, \ldots, T$;

- $\Pi=\left(\Gamma, \mu, R_{\Pi}\right)$ is the skeleton of an extended $\mathrm{P}$ system with active membranes of degree $q$;

- For each $r \in R_{\Pi}$ and for each $j, 1 \leq j \leq m, f_{r, j}$ is a computable function whose domain is $\{1,2, \ldots, T\}$ and its range is contained in $[0,1]$;

- $\Pi_{j}=\left(\Gamma, \mu, R_{\Pi}, T,\left\{f_{r, j}: r \in R_{\Pi}\right\}, \mathcal{M}_{0 j}, \ldots, \mathcal{M}_{q-1, j}\right), 1 \leq j \leq m$, is a probabilistic functional extended $\mathrm{P}$ system with active membranes of degree $q \geq 1$ taking $T$ time units. We denote by $R_{\Pi_{j}}$ the set $R_{\Pi}$ where each rule $r \in R_{\Pi}$ has associated the probabilistic function $f_{r, j}$.

A multienvironment probabilistic functional extended $\mathrm{P}$ system with active membranes of degree $(q, m)$ taking $T$ time units

$$
\left(\Gamma, \Sigma, G, R_{E}, \Pi,\left\{f_{r, j}: r \in R_{\Pi}, 1 \leq j \leq m\right\},\left\{M_{i j}: 0 \leq i \leq q-1,1 \leq j \leq m\right\}\right)
$$

can be viewed as a set of $m$ environments $e_{1}, \ldots, e_{m}$ linked between them by the arcs from the directed graph $G$, as it is represented in the next figure: 


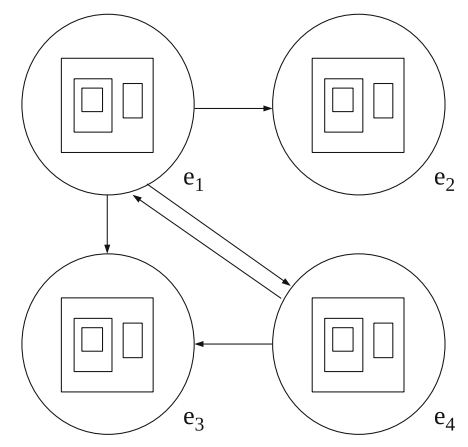

Each environment $e_{j}$ contains a probabilistic functional extended $\mathrm{P}$ system with active membranes of degree $q, \Pi_{j}$, each of them with the same skeleton, $\Pi$, and such that $\mathcal{M}_{0 j}, \ldots, \mathcal{M}_{q-1, j}$ describe their initial multisets.

When a communication rule between environments $(x)_{e_{j}} \stackrel{p_{\left(x, j, j^{\prime}\right)}}{\longrightarrow}(y)_{e_{j^{\prime}}}$ is applied, object $x$ pass from $e_{j}$ to $e_{j^{\prime}}$ possibly modified into an object $y$. In any moment $a, 1 \leq a \leq T$, at which an object $x$ is in environment $e_{j}$, rule will be applied according to its probability which is given by $p_{\left(x, j, j^{\prime}\right)}(a)$, that is, if $\left\{e_{j 1}, \ldots, e_{j z}\right\}$ is the set of reachable nodes from $e_{j}$, and $k$ is the number of objects $x$ in the environment $e_{j}$, then these objects are distributed among the environment $\left\{e_{j 1}, \ldots, e_{j z}\right\}$ according the probabilities $p_{\left(x, j j_{1}\right)}(a), \ldots, p_{\left(x, j, j_{z}\right)}(a)$.

We assume that a global clock exists, marking the time for the whole system (for its compartments), that is, all membranes and the application of all rules are synchronized.

The $(m+m q)$-tuple $\left(E_{1}, M_{01}, \ldots, M_{q-1,1}, \ldots E_{m}, M_{0 m}, \ldots, M_{q-1, m}\right)$ of multisets of objects present at any moment in the $m$ environments and in each of the regions of the $\mathrm{P}$ systems located in the respective environment constitutes a configuration of the system at that moment. The initial configuration of the system is $\left(\emptyset, \mathcal{M}_{01}, \ldots, \mathcal{M}_{q-1,1}, \ldots \emptyset\right.$, $\left.\mathcal{M}_{0 m}, \ldots, \mathcal{M}_{q-1, m}\right)$.

The $\mathrm{P}$ system can pass from one configuration to another by using the rules from $R=R_{E} \cup \bigcup_{j=1}^{m} R_{\Pi_{j}}$ as follows: at each transition step, the number of times applicable rules are applied is selected according to the probabilities assigned to them, and all applicable rules are simultaneously applied and all occurrences of the left-hand side of the rules are consumed, as usual.

\section{A software application}

The simulator developed provides two operating modes, each of them addressed to a specific user type: the ecologist and the designer. The program allows several types of actions for each user category.

On the one hand, the ecologist user is the end-user of the application. This user does not need to know anything about membrane computing, and the program behaves as a black box for him. The goal of the ecologist user is to develop virtual experiments on the ecosystem and, for this purpose, the program allows the next actions:

- Edition of the initial parameters of the ecosystem.

- Selection of the number of years to simulate.

- Selection of the number of simulations per year. 
- To save and load the initial parameters in files.

- To launch simulations.

- Interactive generation of result graphics.

- To save graphics of the results in files.

With these actions, the ecologist user can modify the initial conditions of the ecosystem and run simulations, and then collect the results interactively in graphics files that represent the different populations of species or the amount of biomass produced. For each simulated year, a number of simulations are produced and the average values as well as the standard deviation are saved. The graphics are saved in png format and the initial parameters of the ecosystem are saved in binary files with .ec2 extension. In Fig. 1, the graphics user interface is shown.

Transparently to the ecologist user, the program instantiates in each simulation a $\mathrm{P}$ system exibiting the behavior of the ecosystem with the initial parameters entered.

The designer user is responsible for specifying, debugging and validating the family of $\mathrm{P}$ systems used by the program. We can say that validation is performed experimentally, by comparing simulation results with actual data obtained from the ecosystem. Each simulated year corresponds to a number of computational steps of the $\mathrm{P}$ system, with this information also entered by the designer user.

The specification of the family of $\mathrm{P}$ systems is written in a P-Lingua file which is a programming language to define P systems (García-Quismondo et al. 2009). These files have .pli extension and it is possible to get more information about the P-Lingua project on its website (http://www.p-lingua.org).

The program offers the designer the same actions as to the ecologist, plus some additional actions to facilitate the debugging process:

- Edition of P-Lingua files.

- Compilation of P-Lingua files.

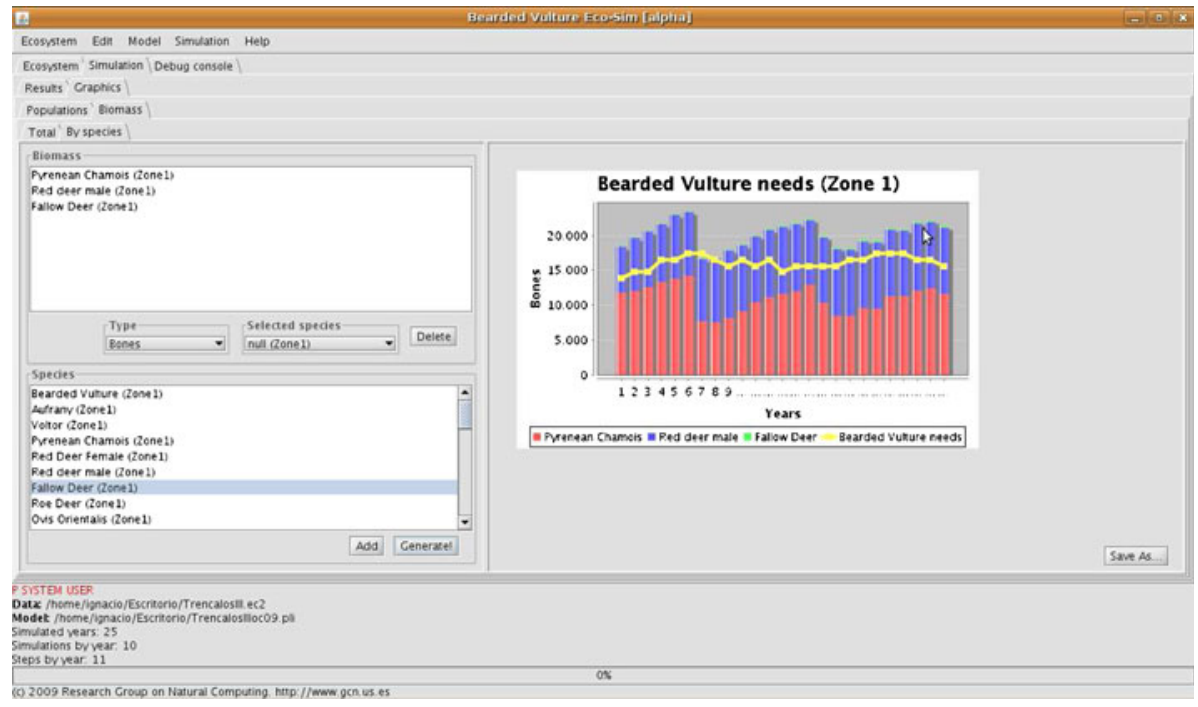

Fig. 1 Graphics user interface 
- Simulation step by step.

- Selection of the number of computation steps per year.

To switch between the two modes of operation, it is necessary to edit a text file called config.cnf.

The application is cross-platform and it has been developed in Java programming language along with the Swing graphical environment by using the following libraries:

- pLinguaCore (http://www.p-lingua.org/wiki/index.php/PLinguaCore), which is responsible for processing P-Lingua files and performing simulations.

- Colt (http://acs.lbl.gov/ hoschek/colt/), which is responsible for generating random numbers.

- JDom (http://www.jdom.org/) for processing XML files.

- JFreeChart (http://www.jfree.org/jfreechart/), which is responsible for generating the graphics after each simulation.

The pLinguaCore library implements a simulation algorithm for probabilistic $\mathrm{P}$ systems based on the binomial distribution, providing a good efficiency. Next, we describe how this algorithm simulates a computation for a finite number of steps.

(a) Rules are classified into sets so that all the rules belonging to a class have the same left-hand side. Note that rules with common objects in their left-hand sides but not exactly the same left-hand side are classified in different sets.

(b) Let $F(N, p)$ be a function that returns a discrete random number within the binomial distribution $B(N, p)$

(c) For each step of simulation do

- Consider a random order on the family of all sets of rules with the same left-hand side.

- For each set of rules $\left\{r_{1}, \ldots, r_{t}\right\}$ whose common left-hand side is $u[v]_{i}^{\alpha}$ and their respective probabilistic constants are $c_{r_{1}}, \ldots, c_{r_{t}}$, according to the order considered, do

* Select a random order on the rules $\left\{r_{1}, \ldots, r_{t}\right\}$

* Compute the highest number $N$ such that $u^{N}$ appears in the father membrane of $i$ and $v^{N}$ appears in membrane $i$.

$* d \longleftarrow 1$

* For each $k(1 \leq k \leq t-1)$, according to the order selected, do

$$
\begin{aligned}
& c_{r_{k}} \longleftarrow \frac{c_{r_{k}}}{d} ; \\
& n_{r_{k}} \longleftarrow\left(N, c_{r_{k}}\right) ; \\
& N \longleftarrow N-n_{r_{k}} ; \\
& q \longleftarrow 1-c_{r_{k}} ; \\
& d \longleftarrow d * q ; \\
& * n_{r_{t}} \longleftarrow N
\end{aligned}
$$

- Each rule $r$ is applied $n_{r}$ times.

The software application is under GNU GPL license that allows free use and modification under some conditions. To obtain a copy of the program or source code, you can contact the authors of the present paper. 


\section{Scavenger birds}

In paper Cardona et al. (2009a), it was presented the first modeling of an ecosystem related to the bearded vulture in the Catalan Pyrenees by using $\mathrm{P}$ systems. The results obtained were quite encouraging. The model was validated using real data of fourteen years. For that period of time, the adjustment was quite good although the model showed an exponential growth of the populations size. So that, it was not suitable for long term prediction. There were also space, food, and other limitations that model in Cardona et al. (2009a) did not take into account. In a second version of the model (Cardona et al. 2009b), new ingredients were incorporated with the aim to overcome some of these limitations. This cell-like model has property only has one environment, and can be considered as a particular case of a multienvironment probabilistic functional extended $\mathrm{P}$ system with active membranes of degree $(2,1)$, where only two polarizations were used, neutral and positive. The ecosystem is composed of 13 species which involved 18 types of animals mainly as a consequence of the management of domestic species.

Paper Cardona et al. (2009a) focused on the modeling of a species of scavenger birds, more specifically on the bearded vulture. In Cardona et al. (2009b), two other species of scavenger birds such as the egyptian and the griffon vultures, which compete against each other for space and food, were added. The bearded vulture feeds on bones and the other two scavenger species on the meat of dead animals. An important source of feeding for these species consists of the domestic animals which die on the country, and for that reason goats, bovines and horses were added in Cardona et al. (2009b). It was taken into account that not all the domestical animals live in the mountain for the whole year.

The source of feeding provided by domestic animals seems to have an end because a regulating police will forbid to leave dead domestic animals on the country. This fact will probably entail an important change in the evolution direction of the ecosystem which will have to be compensated or controled by means of external contributions of food and a good management. The problem presented in Cardona et al. (2009b) allows ecologists to study the evolution of an ecosystem under different scenarios.

The P system presented in Cardona et al. (2009b) is composed of 5 modules and 4 types of different configurations. The annual evolution of the ecosystem is obtained by running the 4 configuration types sequentially. 48 types of rules are used, the number of biological parameters needed for each type of animal is 24 and they are parameters related to reproduction, mortality, feeding and other general ones of the species itself.

The final result is a model with a behavior very close to that obtained from real data, as we can see in Fig. 2. In all cases, real values are in a confidence interval of $95 \%$, marked in the figure by a broken line. Currently, the model and software designed are already useful tools to decide on the management of the ecosystem. The model can be of course improved by incorporating new ingredients which are less important but still of relevance for an almost perfect approach to reality.

\section{Zebra mussel}

Whereas the bearded vulture is an endangered species, the zebra mussel is an invading species. So that the aim of the modeling of the ecosystem is to learn about the evolution of the species with the purpose of introducing management and control mesures of the ecosystem which will decrease or erradicate the species. 

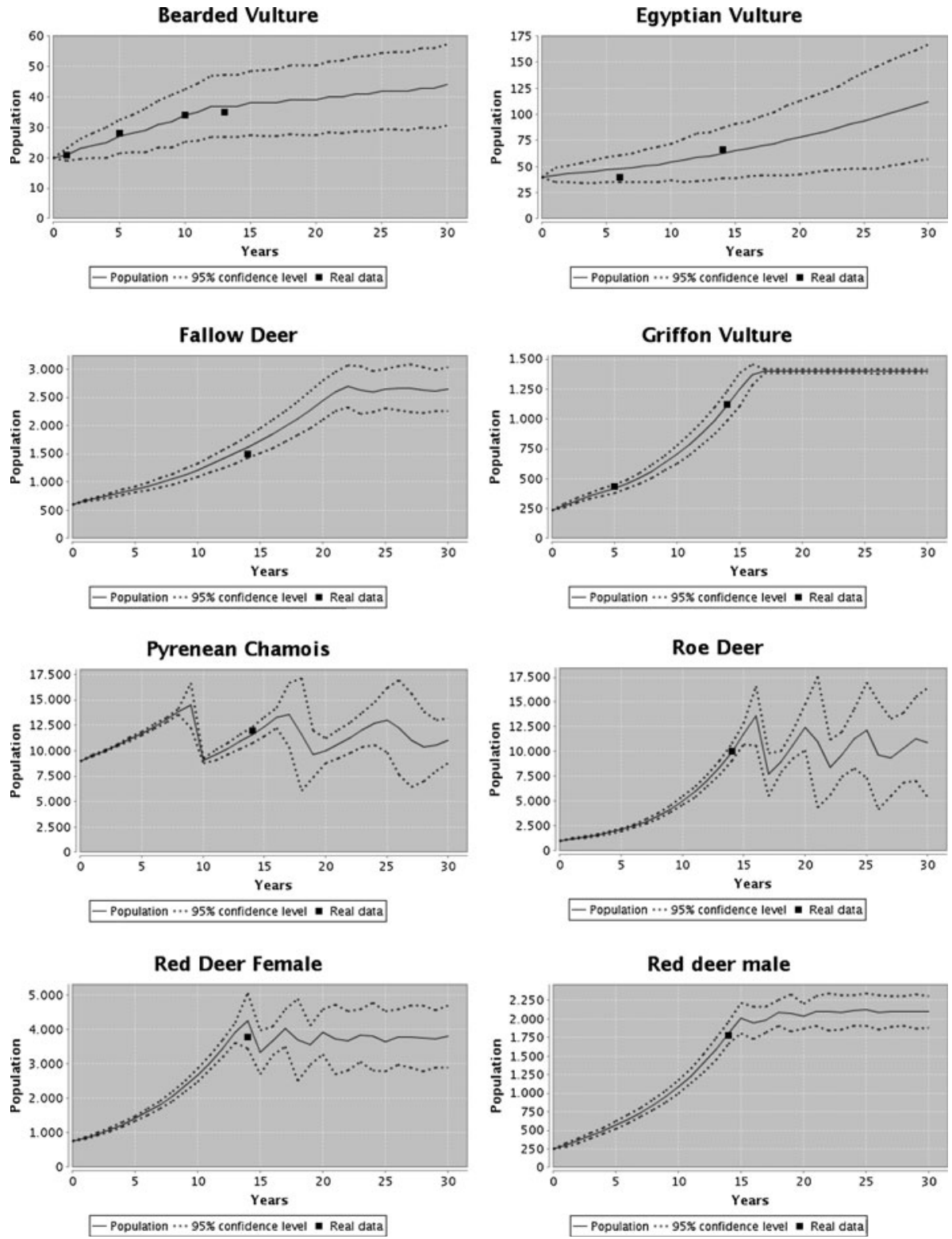

Fig. 2 Experimental validation

In this paper, it is presented a first model which has not been validated with experimental data yet. It has been developed a simulator which allows us to know the number of larvae at each moment as well as the number of adult mussel at the end of the year.

The zebra mussel has an average life expectancy of 3 years (Palau et al. 2003), with a sex proportion acceptable at 1:1. Reproduction takes place when water temperature reaches $15^{\circ} \mathrm{C}$ approximately. 
In the first step, the veliger larva is produced and it remains in the water column growing for a period of 15-28 days (Jenner et al. 1998). After this period, they will get fixed to the substratum. Throughout the year, the zebra mussel has two reproductive cycles. The most important cycle is the first one. First, larvae born along Spring can reach sexual maturity before the second reproductive cycle begins. Therefore, they can reproduce in the second cycle, which takes place at the end of the summer. The number of descendants depends on the age of the adult mussel.

Mussels may stick to each other forming thick agglomerations. When they exceed densities of 250,000 individuals per $\mathrm{m}^{2}$, mussels which are at the bottom of the agglomeration die because they cannot obtain neither nutrients nor other fundamental elements such as oxygen from the environment.

In this work, we model the ecosystem of Ribarroja reservoir which is located in the Northeast region of Spain. It is in a natural environment where there are abundant and varied species and where leisure fishing is one of the main activities. Its length is approximately $28 \mathrm{Km}$, the maximum depth is $28 \mathrm{~m}$. at the foodgate and the minimum is found at the point where the Segre river flows into the reservoir, falling down to $2 \mathrm{~m}$. Its capacity is of $210 \mathrm{Hm}^{3}$.

The presence of zebre mussel was detected in the reservoir at mid-year in 2001 (Ruíz Altaba et al. 2001). The quick expansion of the mussel has led to carrying out of numerous studies, so that at the current moment, there are comprehensive experimental data that allow us to model the ecosystem of the mussel zebra in the reservoir.

The reservoir receives water from the other reservoir and from three rivers. Warmer, superficial waters come from these rivers while colder, deep ones, come from the reservoir. Studies made have come to the conclusion that 17 well differentiated areas can be studied on the basis of temperature fluctuations. All this causes the biological cycle to begin at different moments in the defined areas.

The substratum on which mussels can fix are different according to the area, so that, the densities of mussels are variable in the whole reservoir. If a mussel falls down on a sandy surface it cannot survive. In case it is fixed on other adult mussels and the thickness of the wall formed is high, it may provoke the death of the mussels occupying low layers.

The biology and development of mussels in the reservoir is very complex so that, in this first work, we only model the processes of reproduction, mortality, fixation of the mussel on the substratum, the movement of larvae along the water column and control of densities. Water temperature is also an essential aspect for the development of mussels, and it is taken into account in this modeling. About the complexity of the problem to be modeled, it must be added the effect of the human intervention in the annual management of the reservoir which provokes variations in the movement of larvae among areas.

The model proposed, $M E$, consists of the following multienvironment probabilistic functional extended $\mathrm{P}$ system with active membranes of degree $(5,17)$ taking $T$ time units:

$$
\left(\Gamma, \Sigma, G, R_{E}, \Pi,\left\{f_{r, j}: r \in R_{\Pi}, 1 \leq j \leq 17\right\},\left\{M_{i j}: 0 \leq i \leq 4,1 \leq j \leq 17\right\}\right)
$$

where:

- $\Gamma=\left\{X_{s, c, a}, Y_{s, c, a}: 1 \leq s \leq 6,1 \leq c \leq 2,1 \leq a \leq T\right\} \cup\left\{W_{d, c, a}, Q_{d, c, a}: 0 \leq d \leq 189,1 \leq c\right.$ $\leq 2,1 \leq a \leq T\} \cup\left\{W E_{c, a, s}: 1 \leq c \leq 2,1 \leq a \leq T, 1 \leq s \leq 17\right\} \cup\left\{L_{d c}: 0 \leq d \leq 28,1 \leq c\right.$ $\leq 2\} \cup\left\{t_{i}, t_{i}^{\prime}: 1 \leq i \leq 3\right\} \cup\left\{D_{c d f}: 1 \leq c \leq 2,1 \leq d \leq 14,1 \leq f \leq 7\right\} \cup\left\{D_{c}: 1 \leq c \leq 2\right\}$ $\cup\left\{a_{s}: 0 \leq s \leq 5\right\} \cup\left\{c_{i}: 1 \leq i \leq 2\right\} \cup\left\{e_{i}: 1 \leq i \leq 6\right\} \cup\left\{f_{i}: 1 \leq i \leq 5\right\} \cup\{t, b, M, e, f\}$ $\cup\left\{\eta_{i}: 0 \leq i \leq 153\right\}$ 
Symbols $X, Y$ are associated with different stadiums of mussels. $L$ is associated with larvae and $W, W E$ and $Q$ with mussels born in the present cycle. Object $M$ means that a mussel dies. Objects $D$ allow us to know when temperature reaches the temperature at which reproduction starts. Objects $t_{i}$ signal the start of reproduction in membrane $i$. Object $a_{s}$ allows us to control the density of mussels. Object $c_{i}$ denotes the current status being in the cycle $c_{i}$.

- The objects that can be present in the environment are

$$
\Sigma=\left\{W E_{c, a, j}, W_{1, c, a}: 1 \leq c \leq 2,1 \leq a \leq T, 1 \leq j \leq 17\right\}
$$

- The graph is $G=(V, S)$, where $V=\left\{e_{1}, \ldots, e_{17}\right\}$ and the arcs are $(v, v)$, for each $v \in V$, plus all those depicted in the next figure:

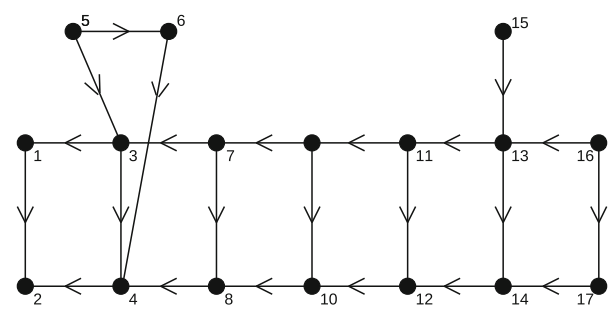

- The set $R_{E}$ of rules of the environment consist of:

$$
\begin{aligned}
& r_{22, c, a, s} \equiv\left(W E_{c, a, s}\right)_{e_{j}} \rightarrow\left(W_{1, c, a}\right)_{e_{s}}, 1 \leq c \leq 2,1 \leq a \leq T, 1 \leq s \leq 17,1 \leq j \leq 17, j \neq s \\
& r_{23, c, a, j} \equiv\left(W E_{c, a, j}\right)_{e_{j}} \rightarrow\left(W_{1, c, a}\right)_{e_{j}}, 1 \leq c \leq 2,1 \leq a \leq T, 1 \leq j \leq 17
\end{aligned}
$$

- $\Pi=\left(\Gamma, \mu, R_{\Pi}\right)$ is a probabilistic functional $\mathrm{P}$ system skeleton with polarization of degree 5 , where $\mu=\left[[]_{1}[]_{2}[]_{3}[]_{4}\right]_{0}$.

- $\Pi_{j}=\left(\Gamma, \mu, R_{\Pi_{j}}, T,\left\{f_{r, j}: r \in R_{\Pi}\right\}, \mathcal{M}_{0 j}, \ldots, \mathcal{M}_{q-1, j}\right), 1 \leq j \leq 17$, is a probabilistic functional extended $\mathrm{P}$ system with active membranes of degree 5 taking $T$ time units.

- For each $r, r \neq 20,21$, and for each $j, 1 \leq j \leq 17, f_{r, j}$ is a computable function defined by $f_{r, j}(a)=k_{r, j}$, for each $a=1,2, \ldots, T$, and $k_{r, j}$ is a real number between 0 and 1, associated with $r$ and $j . f_{r_{20}, j}(a)=F(\varphi(a), j, s)$, where $\varphi(a)$ is the type of management of the reservoir chosen each year, $a(a=1,2, \ldots, T)$, and $F(x, y, z)$ is a real number between 0 and 1 providing the probability of exchange of larvae among environments.

$f_{r_{21}, j}(a)=F(\varphi(a), j, 0)$ provides the probability that larvae go out of the $\mathrm{P}$ system.

- The set $R_{\Pi_{j}}$ of rules of the system consists of:

$$
r_{1} \equiv\left[\eta_{i}\right]_{0}^{0} \rightarrow\left[\eta_{i+1}\right]_{0}^{0}, 0 \leq i \leq 151, i \neq 103, i \neq 136
$$

(At the moment 103 and 136 a synchronization process is produced)

Simulation of temperatures. The sign to activate the cycle of reproduction is obtained when the suitable temperature is reached, therefore it is necessary to simulate the temperature of the water. It is possible to consider that there are three intervals, so that, when temperature reaches the lowest, a percentage of adult mussels initiate reproduction, in the second interval another percentage will do that, and when the highest temperatures are reached the rest of mussels in reproductive age initiate reproduction. The rules to apply are: 


$$
\begin{aligned}
& r_{2 c} \equiv\left[D_{c} \stackrel{f_{r_{2 c}, j}}{\longrightarrow} \lambda\right]_{0}^{0}, 1 \leq c \leq 2,1 \leq j \leq 17 \\
& r_{3 c} \equiv\left[D_{c} \stackrel{1-f_{r_{2 c} \cdot j}}{\longrightarrow} D_{c, 1,1}\right]_{0}^{0}, 1 \leq c \leq 2,1 \leq j \leq 17 \\
& r_{4 c d f} \equiv\left[D_{c, d, f} \stackrel{f_{r_{4 c f 3}, j}}{\longrightarrow} t_{1}, t_{2}, t_{3}\right]_{0}^{0}, 1 \leq d \leq 14,1 \leq f \leq 7,1 \leq c \leq 2,1 \leq j \leq 17 \\
& r_{5 c d f} \equiv\left[D_{c, d, f} \stackrel{f_{r_{s c f 2}, j}}{\longrightarrow} t_{1}, t_{2}, D_{c, d+1, f}\right]_{0}^{0}, 1 \leq d \leq 14,1 \leq f \leq 7,1 \leq c \leq 2,1 \leq j \leq 17 \\
& r_{6 c d f} \equiv\left[D_{c, d, f} \stackrel{f_{r_{6 c f 1}, j}}{\longrightarrow} t_{1}, D_{c, d+1, f}\right]_{0}^{0}, 1 \leq d \leq 14,1 \leq f \leq 7,1 \leq c \leq 2,1 \leq j \leq 17 \\
& r_{7 c d f} \equiv\left[D_{c, d, f} \stackrel{1-\sum_{i=4}^{i=7} f_{r_{i c f}(7-i), j}}{\longrightarrow} D_{c, d+1, f}\right]_{0}^{0}, 1 \leq d \leq 14,1 \leq f \leq 7,1 \leq c \leq 2,1 \leq j \leq 17 \\
& r_{8 c f} \equiv\left[D_{c, 14, f} \stackrel{f_{r_{8 c f 3}, j}}{\longrightarrow} t_{1}, t_{2}, t_{3}\right]_{0}^{0}, 1 \leq f \leq 7,1 \leq c \leq 2,1 \leq j \leq 17 \\
& r_{9 c f} \equiv\left[D_{c, 14, f} \stackrel{f_{r_{c f f 2}, j}}{\longrightarrow} t_{1}, t_{2}, D_{c 1 f+1}\right]_{0}^{0}, 1 \leq f \leq 7,1 \leq c \leq 2,1 \leq j \leq 17 \\
& r_{10 c f} \equiv\left[D_{c, 14, f} \stackrel{f_{r_{10 c f 1, j}}}{\longrightarrow} t_{1}, D_{c, 1, f+1}\right]_{0}^{0}, 1 \leq f \leq 7,1 \leq c \leq 2,1 \leq j \leq 17 \\
& r_{11 c f} \equiv\left[D_{c, 14, f} \stackrel{1-\sum_{i=8}^{i=10} f_{\left.r_{\text {icf }(11-i)}\right)^{j}}^{\longrightarrow}}{\longrightarrow} D_{c, 1, f+1}\right]_{0}^{0}, 1 \leq f \leq 7,1 \leq c \leq 2,1 \leq j \leq 17 \\
& r_{12} \equiv t_{m}[]_{m}^{0} \rightarrow[t]_{m}^{+}, 1 \leq m \leq 3 \\
& r_{13} \equiv[t]_{m}^{+} \rightarrow t[]_{m}^{0}, 1 \leq m \leq 3
\end{aligned}
$$

Process of Reproduction. The process of reproduction takes place in membranes 1, 2 and 3. Inside these membranes there can be objects $X_{s, c, a}$ and $Q_{d, c, a}$ that represent adult mussels and mussels born in the previous cycle respectively.

$$
\begin{aligned}
r_{14} & \equiv\left[Q_{d, c, a}\right]_{m}^{+} \stackrel{0.5}{\longrightarrow} Y_{1, c, a} L_{0, c, a}^{g_{c, 1}}[]_{m}^{0}, 1 \leq a \leq T, n(j) \leq d \leq 180,1 \leq c \leq 2,1 \leq m \leq 3,1 \leq j \leq 17 \\
r_{15} & \equiv\left[Q_{d, c, a}\right]_{m}^{+} \stackrel{0.5}{\longrightarrow} Y_{1, c, a}[]_{m}^{0}, 1 \leq a \leq T, n(j) \leq d \leq 180,1 \leq c \leq 2,1 \leq m \leq 3,1 \leq j \leq 17 \\
r_{16} & \equiv\left[Q_{d, c, a}\right]_{m}^{+} \rightarrow Y_{1, c, a}[]_{m}^{0}, 1 \leq a \leq T, 0 \leq d<n(j), 1 \leq c \leq 2,1 \leq m \leq 3,1 \leq j \leq 17 \\
r_{17} & \equiv\left[X_{s, c, a}\right]_{m}^{+} \stackrel{0.5}{\longrightarrow} Y_{s, c, a} L_{0, c, a}^{g_{c, s}}[]_{m}^{0}, 1 \leq a \leq T, 1 \leq s \leq 6,1 \leq c \leq 2,1 \leq m \leq 3 \\
r_{18} & \equiv\left[X_{s, c, a}\right]_{m}^{+} \stackrel{0.5}{\longrightarrow} Y_{s, c, a}[]_{m}^{0}, 1 \leq a \leq T, 1 \leq s \leq 6,1 \leq c \leq 2,1 \leq m \leq 3
\end{aligned}
$$

Larvae growth. Growing larvae can stay in the environment where they were born or can move to other ones.

$$
\begin{aligned}
r_{19} & \equiv\left[L_{d, c, a} \rightarrow L_{d+1, c, a}\right]_{0}^{0}, 0 \leq d<28,1 \leq a \leq T, 1 \leq c \leq 2 \\
r_{20} & \equiv\left[L_{28, c, a}\right]_{0}^{0} \stackrel{f_{r_{20}, j}}{\longrightarrow} W E_{c, a, s}[]_{0}^{0}, 1 \leq c \leq 2,1 \leq a \leq T, 1 \leq s \leq 17,1 \leq j \leq 17 \\
r_{21} & \equiv\left[L_{28, c, a} \stackrel{f_{r_{21}, j}}{\longrightarrow} \lambda\right]_{0}^{0}, 1 \leq c \leq 2,1 \leq a \leq T, 1 \leq j \leq 17
\end{aligned}
$$

Larvae entering the environment

$$
r_{24} \equiv W_{1, c, a}[]_{0}^{0} \rightarrow\left[W_{2, c, a}\right]_{0}^{0}, 1 \leq c \leq 2,1 \leq a \leq T
$$

Mortality associated with senescence.

$$
r_{25} \equiv\left[Y_{6, c, a} \rightarrow M\right]_{0}^{0}, 1 \leq c \leq 2,1 \leq a \leq T
$$


All mussels must enter membrane 4.

$$
\begin{aligned}
r_{26} & \equiv Y_{s, c, a}[]_{4}^{0} \rightarrow\left[Y_{s, c, a}\right]_{4}^{0}, 1 \leq s \leq 5,1 \leq c \leq 2,1 \leq a \leq T \\
r_{27} & \equiv W_{2, c, a}[]_{4}^{0} \rightarrow\left[Q_{3, c, a}\right]_{4}^{0}, 1 \leq c \leq 2,1 \leq a \leq T \\
r_{28} & \equiv\left[Q_{d, c, a} \rightarrow Q_{d+1, c, a}\right]_{4}^{0}, 3 \leq d \leq 180,1 \leq c \leq 2,1 \leq a \leq T
\end{aligned}
$$

Reproduction cycle does not take place. In case the conditions to start the reproductive cycle are not produced, the following rules allow us to continue the process.

$$
\begin{aligned}
r_{29} & \equiv\left[\eta_{103} \rightarrow t_{1}^{\prime} t_{2}^{\prime} t_{3}^{\prime} \eta_{104}\right]_{0}^{0} \\
r_{30} & \equiv t_{m}^{\prime}[]_{m}^{0} \rightarrow[t]_{m}^{-}, 1 \leq m \leq 3 \\
r_{31} & \equiv\left[X_{s, c, a}\right]_{m}^{-} \rightarrow Y_{s, c, a}[]_{m}^{0}, 1 \leq s \leq 6,1 \leq c \leq 2,1 \leq a \leq T, 1 \leq m \leq 3 \\
r_{32} & \equiv\left[Q_{d, c, a}\right]_{m}^{-} \rightarrow Y_{1, c, a}[]_{m}^{0}, 1 \leq d \leq 180,1 \leq c \leq 2,1 \leq a \leq T, 1 \leq m \leq 3 \\
r_{33} & \equiv[t]_{m}^{-} \rightarrow t[]_{m}^{0}, 1 \leq m \leq 3
\end{aligned}
$$

Mussel viability associated to population density. In this process, it is analyzed the viability of young and adult mussels. For the first ones, viability depends on the type of substratum and for the last ones it depends on their position.

$$
\begin{aligned}
& r_{34} \equiv \eta_{138}[]_{4}^{0} \rightarrow \eta_{139}[b]_{4}^{+} \\
& r_{35} \equiv[h]_{4}^{+} \rightarrow b\left[a_{0}^{B(k) * 250000}\right]_{4}^{0}, 1 \leq k \leq 17 \\
& r_{36} \equiv\left[a_{s}\right]_{4}^{+} \rightarrow b\left[a_{s+1}\right]_{4}^{0}, 0 \leq s \leq 4 \\
& r_{37} \equiv[e]_{4}^{+} \rightarrow b\left[f_{1}\right]_{4}^{0} \\
& r_{38} \equiv\left[e_{i}\right]_{4}^{+} \rightarrow b\left[f_{i}\right]_{4}^{0}, 1 \leq i \leq 5 \\
& r_{39} \equiv\left[f_{i}\right]_{4}^{0} \rightarrow b\left[e_{i+1}\right]_{4}^{+}, 1 \leq i \leq 5 \\
& r_{40} \equiv\left[e_{6}\right]_{4}^{+} \rightarrow b[f]_{4}^{0} \\
& r_{41} \equiv\left[Q_{d, c, a} a_{0}\right]_{4}^{0} \rightarrow Q_{d+1, c, a}[]_{4}^{+}, 1 \leq a \leq T, 3 \leq d \leq 180,1 \leq c \leq 2 \\
& r_{42} \equiv\left[Y_{s, c, a} a_{s}\right]_{4}^{0} \rightarrow X_{s+1, c+(-1)^{c+1, a}}[]_{4}^{+}, 1 \leq a \leq T, 1 \leq s \leq 5,1 \leq c \leq 2 \\
& r_{43 m} \equiv X_{s, c, a}[]_{m}^{0} \stackrel{f_{r_{43 m}, j}}{\longrightarrow}\left[X_{s, c, a}\right]_{m}^{0}, 1 \leq a \leq T, 1 \leq m \leq 3,1 \leq c \leq 2,1 \leq j \leq 17 \\
& r_{44 m} \equiv Q_{d, c, a}[]_{m}^{0} \stackrel{f_{r_{44 m}, j}}{\longrightarrow}\left[Q_{d+1+(c-1) * 60, c+(-1)^{c+1}, a+(c-1)}\right]_{m}^{0}, 1 \leq a \leq T, 0 \leq d \leq 121 \text {, } \\
& 1 \leq m \leq 3,1 \leq c \leq 2,1 \leq j \leq 17 \\
& r_{45} \equiv\left[Q_{d, c, a} \rightarrow Q_{d+1, c, a}\right]_{m}^{0}, 1 \leq a \leq T, 5 \leq d \leq 121,1 \leq c \leq 2,1 \leq m \leq 3
\end{aligned}
$$

Preparation for the beginning of the new cycle. Elimination of created objects and reinitialization of the counters. 


$$
\begin{aligned}
r_{46} & \equiv \eta_{152}[]_{4}^{0} \rightarrow \eta_{153}[h]_{4}^{-} \\
r_{47} & \equiv\left[Y_{s, c, a}\right]_{4}^{-} \rightarrow M[]_{4}^{0}, 1 \leq s \leq 5,1 \leq c \leq 2,1 \leq a \leq T \\
r_{48} & \equiv\left[Q_{d, c, a}\right]_{4}^{-} \rightarrow M[]_{4}^{0}, 1 \leq d \leq 121,1 \leq c \leq 2,1 \leq a \leq T \\
r_{49} & \equiv\left[a_{s}\right]_{4}^{-} \rightarrow b[]_{4}^{0}, 0 \leq s \leq 6 \\
r_{50} & \equiv[b]_{4}^{-} \rightarrow b[]_{4}^{0} \\
r_{51} & \equiv[b \rightarrow \lambda]_{0}^{0} \\
r_{52} & \equiv[t \rightarrow \lambda]_{0}^{0} \\
r_{53} & \equiv\left[\eta_{153} \rightarrow \eta_{0}\right]_{0}^{0} \\
r_{54} & \equiv\left[c_{i}\right]_{4}^{-} \rightarrow D_{i+(-1)^{i+1}}\left[c_{i+(-1)^{i+1}}\right]_{4}^{0}, 1 \leq i \leq 2 \\
r_{55} & \equiv[f]_{4}^{-} \rightarrow b[e]_{0}^{4}
\end{aligned}
$$

- $\mathcal{M}_{0 j}, \mathcal{M}_{1 j}, \mathcal{M}_{2 j}, \mathcal{M}_{3 j}$ and $\mathcal{M}_{4 j}(1 \leq j \leq 17)$ are strings over $\Gamma$ describing the multisets of objects initially placed in regions of $\mu$

$$
\begin{aligned}
\left\{\left(\mathcal{M}_{i j}\right\}_{1 \leq i \leq 3,1 \leq j \leq 17}\right. & =\left\{X_{s, 1,1}^{q_{i j}}: 1 \leq s \leq 6\right\} ; \\
\left\{\left(\mathcal{M}_{4}\right)_{e}\right\}_{1 \leq e \leq 17} & =\left\{h, c_{1}, e\right\} ; \\
\left\{\left(\mathcal{M}_{0}\right)_{e}\right\}_{1 \leq e \leq 17} & =\left\{\eta_{0}, T_{1}\right\} ;
\end{aligned}
$$

The information necessary is the following:

- Quantity of mussels in each zone, $q_{i j}, 1 \leq i \leq 3,1 \leq j \leq 17$.

- Average temperature throughout the year. It's necessary in order to calculate the probabilities associated with the rules $r_{2}$ to $r_{11}$

- Percentage of mussels that initiate the reproductive cycle depending on the temperature, $f_{r 43, m, j}$ and $f_{r 44, m, j}, 1 \leq m \leq 3,1 \leq m \leq 17$.

- Quantity of viable larvae per mussel in function of age, $g_{c, s}, 1 \leq c \leq 2,1 \leq s \leq 6$.

- Probability of larvae moving among environments, $f_{r 20}$ and $f_{r 21}$.

- Maximum density of alive mussels in the area, $250,000 \mathrm{ind} / \mathrm{m}^{2}$.

- Age measured in days in which sexual maturity is reached, $n(j), 1 \leq j \leq 17$.

- Type of soil for area, $B(k), 1 \leq k \leq 17$.

We are working now to write programs for simulating this model so that we can check the model against real data and tune it in view of future applications.

\section{Conclusions and future works}

P systems have been presented as a high level computational modeling framework which integrates the structural and dynamical aspects of cellular systems in a comprehensive and relevant way while providing the required formalization to perform mathematical and computational analysis. This framework has been successfully used to model proteinic signaling pathways (Cheruku et al. 2007), gene expression control (Romero and Pérez-Jiménez 2008b), molecular mechanisms of quorum sensing (Romero and PérezJiménez 2008a), etc. 
In this paper, a $\mathrm{P}$ systems based general framework for modeling ecosystems dynamics is presented. Syntax has been specified by means of multienvironment $\mathrm{P}$ systems and the semantics is of a probabilistic type in a special variant of $\mathrm{P}$ systems with active membranes. Two real ecosystems have been considered as case studies: scavenger birds at the Catalan Pyrenees and the zebra mussel at Ribarroja reservoir (Spain). Unlike the model for scavenger birds, the model of the ecosystem related to the zebra mussel has not been validated yet. For this reason, we are working on the design of a software tool which enables us to run simulations of this model and then validate it experimentally.

Acknowledgement The fifth and the sixth author acknowledges the support of the project TIN2009-13192 of the Ministerio de Ciencia e Innovación of Spain, cofinanced by FEDER funds as well as the support of the Project of Excellence with Investigador de Reconocida Valia of the Junta de Andalucía, grant P08-TIC04200 .

\section{References}

Cardona M, Colomer MA, Pérez-Jiménez MJ, Sanuy D, Margalida A (2009a) Modelling ecosystems using P systems: the bearded vulture, a case of study. Lecture Notes in Computer Science 5391:137-156

Cardona M, Colomer MA, Pérez Hurtado I, Pérez-Jiménez MJ, Sanuy D, Margalida A (2009b) A P system based model of an ecosystem of some scavenger birds. Proceeding of the 10th International Workshop on Membrane Computing, pp 153-168

Cheruku S, Paun A, Romero FJ, Pérez-Jiménez MJ, Ibarra OH (2007) Simulating FAS-induced apoptosis by using P systems. Prog Nat Sci 17(4):424-431

Fontana F, Manca V (2008) Predator-prey dynamics in P systems ruled by metabolic algorithm. BioSystems 91:545-557

García-Quismondo M, Gutiérrez-Escudero R, Martínez-del-Amor MA, Orejuela E, Pérez-Hurtado I (2009) P-Lingua 2.0: a software framework for cell-like P systems. Int J Comput Commun Control 4(3):234243

Jenner HA, Whitehouse JW, Taylos CJL, Khalanski M (1998) Cooling water management in European power stations. Biology and Control of fouling. Hidroecol Appl 10:1-2

Lotka AJ (1925) Elements of physical biology. Reprinted by Dover in 1956 as Elements of Mathematical Biology

Palau A, Cía I, Fargas D, Bardina M, Massuti S (2003) Resultados preliminares sobre ecología básica y distribución del mejillón cebra en el embalse de Riba-Roja (río Ebro). UPH Ebro-Pirineos (ENDESA Generación) Dirección de Medio Ambiente y Desarrollo Sostenible (ENDESA Servicios)

Romero FJ, Pérez-Jiménez MJ (2008a) A model of the Quorum Sensing System in Vibrio Fischeri using P systems. Artif Life 14(1):95-109

Romero FJ, Pérez-Jiménez MJ (2008b) Modelling gene expression control using P systems: the Lac Operon, a case study. BioSystems 91(3):438-457

Ruíz Altaba C, Jiménez PJ, López MA (2001) El temido mejillón cebra empieza a invadir los ríos españoles desde el curso bajo del río Ebro. Quercus 188:50-51

Volterra V (1926a) Variazioni e fluttuazioni del numero d'individui in specie animali conviventi. Mem R Accad Naz dei Lincei 2:31-113

Volterra V (1926b) Fluctuations in the abundance of a species considered mathematically. Nature 118:558560 